\title{
Power Supply Issues in E-health Monitoring Applications
}

\author{
Alan Davidson ${ }^{1}$, Neha Mathur ${ }^{2}$, Ivan Glesk ${ }^{3}$, and Arjan Buis ${ }^{4}$ \\ ${ }^{1,2,3}$ Dept. of Electronic and Electrical Engineering \\ Faculty of Engineering \\ University of Strathclyde \\ 204 George Street, Glasgow, G1 1XW, United Kingdom \\ ${ }^{4}$ Dept. of Biomedical Engineering \\ Faculty of Engineering \\ University of Strathclyde \\ 106 Rottenrow, Glasgow, G1 1XW, United Kingdom
}

\section{Phone number: +44 41548 4978, e-mail: Alan.Davidson@strath.ac.uk, Ivan.Glesk@strath.ac.uk, Neha.Mathur@strath.ac.uk, Arjan.Buis@strath.ac.uk}

\section{Key words.}

e-health, health monitoring, power cycling

\begin{abstract}
Recent years have seen a rapid growth in the development of e-health systems for the continuous remote patient monitoring of physiological data such as temperature, heart rate (HR), Blood pressure (BP), oxygenation $(\mathrm{SpO} 2)$, respiration and glucose levels. The roll-out of such schemes show promise in delivering improvements in patient care while at the same time reducing both the demand for resources and the financial burden on healthcare systems. These wearable monitoring systems are used to monitor, log and transmit patient data to a central health authority. Depending on the patient, it is often critical that the monitoring system reliability is high to deliver the appropriate patient care and ensure patient safety. However, since wearable systems are solely dependent on battery power, continuous monitoring will rapidly deplete the battery energy making the system prone to failure. In this paper, methods to reduce power consumption will be discussed using the example of prosthetic socket temperature monitoring data.
\end{abstract}

\section{Introduction}

The advent of the internet and mobile communications devices together with a public desire for self-health monitoring has spawned the development of a plethora of cheap wearable health monitoring devices for measuring and logging a wide range of parameters such as calories burnt, steps taken, body mass index, $\mathrm{SpO} 2$ and heart $\mathrm{HR}$ to name a few. In addition, smartphone apps for health monitoring are now commonplace with examples including a pedometer and a colour analyzer. In fact, it is estimated that at least $70 \%$ of Americans monitor at least one health indicator with $60 \%$ tracking weight, exercise and diet; while $33 \%$ track quantities such as blood pressure (BP), glucose and sleep patterns [1]. While these devices and apps are aimed at the consumer market, this technology has opened up the possibility of routine remote patient monitoring by health authorities under pressure to curtail rising costs while at the same time delivering improvements in patient welfare [2]. In fact, the continuous monitoring, logging and processing of patient vital signs is feasible using existing technology.

Such systems will allow, for example, remote patient/doctor consultation and the remote monitoring of physiological data and patient vital signs such as temperature, photoplethysmogram (PPG), HR, BP, oxygenation (SpO2), respiration and glucose levels. Such medical monitoring systems may utilise on-body (noninvasive) or in-body sensors and will generally incorporate the basic components of a microcontroller based unit (MCU) for control and pre-processing; and a transmitter and smart-phone for data processing and data transfer via the internet to an e-health server and database.

Data collected can be used to provide an early warning of, for example, serious health threats or patient drug compliance failures. In addition, geographical location and movement patterns can be logged in the case of vulnerable patients such as the elderly or those with dementia. Deviation in normal patterns of behaviour and/or changes in vital signs may be an indicator that something is wrong and the patient data may then be used to trigger medical intervention or an emergency response as appropriate. The early warning of medical complications or non-compliance and emergencies could potentially save lives and help reduce the incidence of costly hospital admission in already overstretched health 
authorities. In addition, the need for scheduled attendance at outpatient clinics and doctor's surgeries could be reduced allowing greater patient freedom and an improved quality of life. Furthermore, continuous monitoring can provide a more realistic indicator of patient health status by providing information that would be otherwise remain unrecorded.

To become ubiquitous, such a monitoring system must be portable, wearable, comfortable, secure, robust and, most critically, reliable in the measurement, logging and communication of data. Lost patient data due to unreliable mobile communications could compromise patient safety while on the other hand false alarms could become costly and tie up limited healthcare resources. While it is clear that false alarms should be avoided as much as possible, this must not be at the cost of patient safety. In addition since many of the patients who would most benefit from such a monitoring system will be the elderly, disabled and infirm it is desirable that the monitoring system should not only require a minimum of user intervention but should be well tolerated by the patient. In particular, it is important to make the system as small and light as possible. Incorporating devices into a ring or watch has been suggested [3] for example.

However issues of concern in the realisation of the above requirements are the power consumption of the device and the longevity of the battery between charges. Ideally the device battery should be able to supply enough charge to power the device for extended periods. However, current battery technology is not delivering the improvements in capacity currently demanded by the development of smaller, more resource hungry devices. Reducing battery size results in poor battery life between charges and the current technology has therefore become the enemy of device miniaturisation. In fact the year on year improvement is relatively slow compared to the increase in power demand with battery capacity estimated to be improving by only $8 \%$ per year [4]. The ubiquitous lithium battery, adopted widely due to it's relatively high safety/capacity characteristics is reaching it's peak. New battery technologies are under investigation to improve energy density, however most of these contain toxic or highly flammable materials - for example hydrogen fuel cells - and are therefore not suitable for human monitoring applications. In short while devices are reducing in size, the battery size cannot be reduced without incurring a severe penalty in the level of charge it can hold. In fact, many wrist-worn smart devices must be charged daily with the best of them requiring to be charged at least every 2 days.

In the absence of adequate battery technologies, it is clearly important to minimize device power consumption and consider possible solutions such as incorporating alternative power sources such as kinetic, heat or solar which may be used to supplement battery charge. Also, the design of devices operating using CMOS technology in the sub-threshold region may provide an answer to for some applications by extending battery life up to 10 fold [5]. However problems exist with process, voltage and temperature range limitations which could adversely affect the key device requirement of reliability. It is however crucial that since monitoring devices are in direct human contact, they must be able to operate across widely varying environmental conditions.

In the meantime, significant reductions in power consumption may be achieved by, for example reducing the duty cycle of sensors, reducing the data sampling rate, reducing the MCU processor clock speed; and reducing the amount of data transmitted between MCU and smartphone and smart-phone and server. However, careful consideration must be made between the relative importance of balancing the conflicting requirements of, on the one hand providing sufficient battery power supply life; and on the other, providing a sufficient level of information to ensure reliable diagnosis and decision making.

In many e-health sensing applications Bluetooth is used for transmitting the data collected from the sensor(s) to smartphone [6]. Bluetooth is a suitable wireless technology for remote health monitoring due to it's inherent simplicity, adaptability and security. In addition it's incorporation is ubiquitous in modern mobile phones and PCs. Bluetooth technology is designed to have very low power consumption and, as such, uses less than 3 percent of the power required by Wi-Fi for the same tasks. For example, sending data at the rate of 75 bytes per second over Wi-Fi requires approximately 80 milliwatts of electrical power. Sending data at the same rate over Bluetooth consumes only 2 milliwatts [7]. However, it is expected that further savings in power consumption can be achieved by applying the techniques described in the following sections.

\section{Limb Skin Temperature}

The core temperature of the human body remains constant throughout the day by a process known as thermoregulation, despite internal heat production and the variation in environmental temperature [8]. The system works on negative feedback control, that is, the thermoreceptors sense an increase in temperature that is analysed by the control mechanism (hypothalamus), which in turn causes a response from the effectors to lose more heat e.g. by vasodilatation of blood vessels or by increasing the perspiration rate. The opposite happens if the thermoreceptors sense a decrease in skin temperature. Sometimes the function of the thermoregulatory system can be altered through disease, environmental extremes and drug usage [9]. This could result in serious complications like infection, gangrene, Charcot foot disease and amputation [10]. It is therefore important to monitor the limb skin temperature to provide an early warning of potentially serious medical problems. A common complaint by many amputees is discomfort caused by elevated temperature and associated perspiration within their prosthetic socket. Furthermore, increased perspiration can heighten the risk of skin maceration, leading to an increase in the risk of infection and the development of potentially serious pressure ulcers. The continuous monitoring of the prosthetic socket 
temperature could therefore aid the prevention of these debilitating conditions. The heat dissipation in prosthetic sockets is greatly influenced by the thermal conductive properties of the socket and interface liner materials. These materials influence the body's temperature regulation mechanism and might be the reason for thermal discomfort in prosthetic sockets.

We have non-invasively monitored the limb skin temperature of a human subject by means of thermocouples placed within the lower limb prosthetic socket [11]. Limb skin and liner/socket interface temperatures were measured at three ambient temperatures $5^{\circ} \mathrm{C}, 15^{\circ} \mathrm{C}$ and $25^{\circ} \mathrm{C}$ as shown in Fig. 1. The sampling rate was fixed at $0.5 \mathrm{~Hz}$ over the various levels of activity. It was observed that the stump skin temperature behaviour is a function of ambient temperature. The data was transmitted via Bluetooth to a mobile smart-phone. However, issues with power consumption have restricted the device usage between charges and therefore questioned the suitability of the existing technology for critical health monitoring applications.

\section{Power Reduction Strategies}

Strategies for reducing power consumption take advantage of opportunities in many areas of system design, for example at the CMOS transistor level or in reducing the duty cycle of the system sensors, reducing the data sampling rate, reducing the MCU processor clock speed; and reducing the amount of data transmitted between MCU and smart-phone and smart-phone and server.

The use of power cycling provides an opening for reducing average power consumption in applications where energy use must be tightly managed. This article focuses on power cycling and its impact on overall power consumption. An important energy management technique, power cycling is the process of removing power from a function when it is not needed, such as shutting off a sensor system when measurements are not required. This enables a reduction in average power dissipation, as quantified by equation (1):

$$
\mathrm{P}_{\mathrm{AV}}=\mathrm{D} \times \mathrm{P}_{\mathrm{ON}}+(1-\mathrm{D}) \times \mathrm{P}_{\mathrm{OFF}}
$$

Where $\mathrm{D}$ is the duty cycle given by $\mathrm{D}=\mathrm{T}_{\mathrm{ON}} /\left(\mathrm{T}_{\mathrm{ON}}+\mathrm{T}_{\mathrm{OFF}}\right)$ where $\mathrm{P}_{\mathrm{ON}}$ is the system's power dissipation in its normal operating state and $\mathrm{P}_{\mathrm{OFF}}$ is the system's power consumption in its OFF state. $\mathrm{P}_{\mathrm{OFF}}$ is associated with residual currents, such as maintaining a power switch or shutdown mode in a power regulator, and typically has a value of the order of $1 \mu \mathrm{A}$. The $\mathrm{ON}$ time $\left(\mathrm{T}_{\mathrm{ON}}\right)$ is the amount of time for the sensor system to turn on, produce a desired measurement, and turn back off. The OFF time $\left(\mathrm{T}_{\mathrm{OFF}}\right)$ depends on how frequently the system requires sensor measurements. If $\mathrm{P}_{\mathrm{OFF}}$ is much smaller than the $\mathrm{ON}$ power, the average power dissipation is almost proportional to the duty cycle. For example, if $\mathrm{P}_{\mathrm{OFF}}$ is zero and the duty cycle is $10 \%$, the average power dissipation is $10 \%$ of the normal operating power.

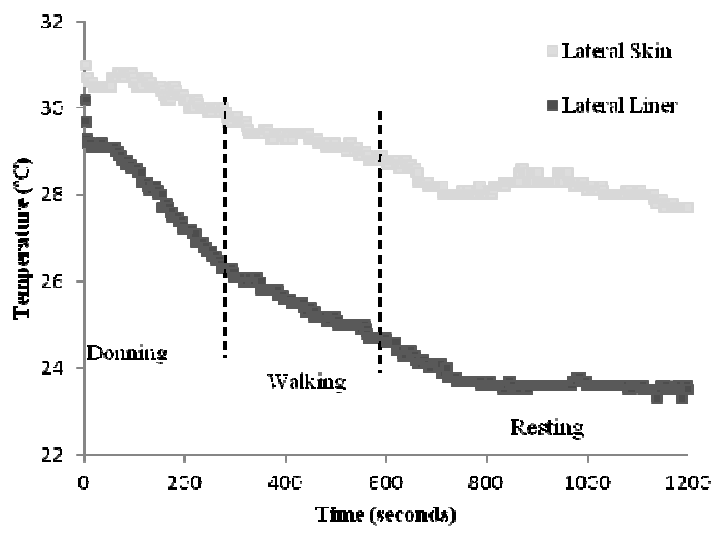

(a)

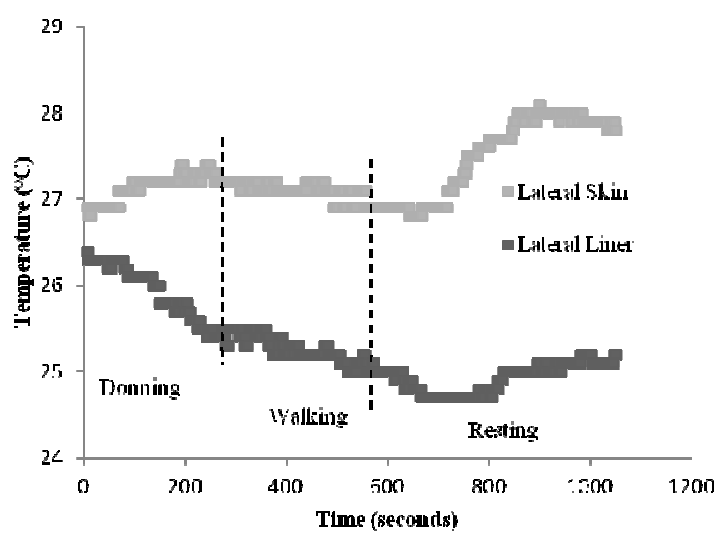

(b)

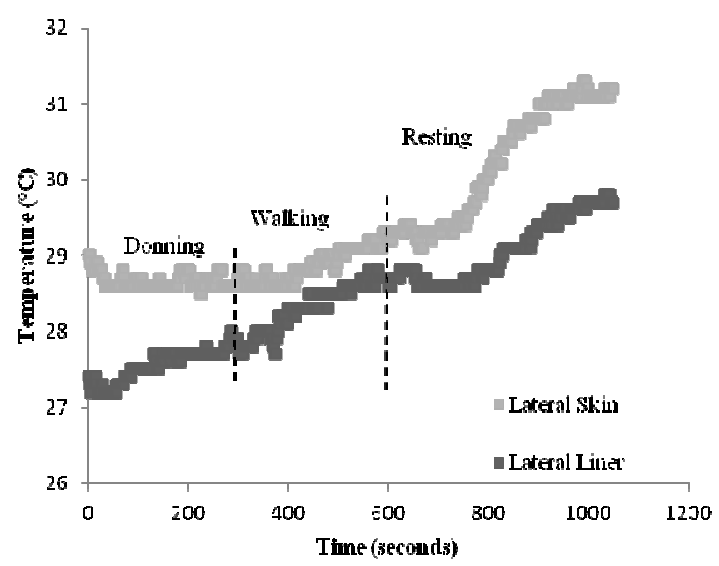

(c)

Fig. 1. Temperature profile of the lateral stump skin and liner at (a) $5^{\circ} \mathrm{C}$; (b) $15^{\circ} \mathrm{C}$; (c) $25^{\circ} \mathrm{C}$ ambient temperatures.

When evaluating the effectiveness of power cycling in a sensor system, the time taken to acquire the data must be known. The duty cycle time is made up of two components, the power ON measurement time and the power OFF time. To determine the duty cycle it is important to consider three different time phases from the initial start up to the data acquisition at the end of the ON phase. $\mathrm{T}_{\mathrm{ON}}$ is dependent on the start-up time, the settling time, and the data-acquisition time. The start-up time depends on the system processor and the initialization routines it must run to support sensor data sampling and additional signal-processing operations. Settling time can include for example the electrical behaviour of 
measurement transducers, interface circuits, filters; and the thermal or mechanical settling time. In some cases, this transient behaviour settles during the start-up time and consequently they have little or no impact on the overall measurement time. However, the prudent approach to analysing the start-up and settling transients is to assume that they happen in cascade, unless it is known that simultaneous startup and settling occurs. The dataacquisition time is dependent on how many data samples are required and the MCU clock speed which affects how fast the MCU can process data.

At the transistor level, the dynamic power consumption of a CMOS device is proportional to the clock frequency and the square of the supply voltage. The well-known relationship: $\mathrm{P}=\mathrm{C} \mathrm{V}_{\mathrm{dd}}^{2} \mathrm{f}$ illustrates this relationship where $\mathrm{P}$ is the transistor dynamic switching power consumption; $\mathrm{C}$ is the CMOS switch lumped capacitance which is the sum of the junction capacitances and gate capacitances; $\mathrm{V}$ is the supply voltage; and $\mathrm{f}$ is the clock frequency. Maintaining $\mathrm{V}_{\mathrm{dd}}$ constant while halving the clock frequency will reduce power consumption by half, however the total energy consumed will be unaffected. This is because the energy consumed is the time integral of power and therefore the energy consumed by an operation will not be reduced since the same number of clock cycles is required to do the same work. However, reducing the clock frequency, f allows $V_{d d}$ to be reduced leading to reduced power consumption. The reduction in $\mathrm{V}_{\mathrm{dd}}$ increases the CMOS delay. However, this is of no consequence if the reduced clock speed is sufficient, that is where all operations required are completed in sufficient time before the next sample. Thus, reducing power consumption and voltage can significantly reduce power and energy consumption. Where this technique is used in conjunction with power cycling, careful consideration must be taken of the likely increase in dataacquisition time and start-up time which will have the consequence of reducing the $\mathrm{P}_{\text {off }}$ time, offsetting the power saving achieved by reducing $f$. In consideration of the settling time, the thermocouple output voltage does not fall during $\mathrm{P}_{\text {off }}$, consequently there is no associated settling time at $\mathrm{P}_{\mathrm{on}}$ using thermocouples. However, signal conditioning circuits will have an associated settling time which will cause an increase in the duty cycle.

Power consumption is also dependent on the sampling rate. The fewer the samples, the shorter can be the duty cycle. In addition fewer samples mean a further power saving through lower data transmission rates. Sampling at a period shorter than the finite response of the thermocouple to temperature transients may result in inaccuracies in measurement in addition to incurring an unnecessary power penalty. To determine the shortest sampling period the thermal response of a thermocouple in the prosthetic socket is considered. For example, when a move from an outside low temperature of $5^{\circ} \mathrm{C}$ to an inside temperature of $25^{\circ} \mathrm{C}$ occurs, sampling faster than the thermocouple transient period for a given temperature change and required accuracy will provide no additional information.
Assuming that the heat transfer between the limb skin and thermocouple obeys Newtonian heat transfer, the heat transfer equation is given by the well-known relationship:

$$
\text { h A }\left(T_{f}-T\right)=m c d T / d t
$$

Where $\mathrm{h}$ is the heat transfer coefficient; $\mathrm{A}$ is the surface area of the thermocouple; $T_{f}$ is the temperature of the limb skin being measured; $\mathrm{T}$ is the temperature of the thermocouple; $\mathrm{m}$ is the mass of the thermocouple; and $\mathrm{c}$ is the heat capacity of the thermocouple. The solution of this first order equation is:

$$
T=T_{f}+\left(T_{i}-T_{f}\right) e^{-h A t / m c}
$$

Where $T_{i}$ is the initial temperature of the thermocouple. It is clear from equation (3) that how quickly $T$ to approaches $T_{f}$ is dependent on the difference between the initial temperature of the thermocouple, $\mathrm{T}_{\mathrm{i}}$ and limb skin, $\mathrm{T}_{\mathrm{f}}$. While it is unlikely that large temperature transients will occur with activity at a constant ambient temperature as indicated in Fig. 1, the dependency of skin temperature on ambient temperature may incur relatively large temperature transients if the subject moves between areas of large temperature differences such as from inside at room temperature to outside on a cold winter day. Since such behaviour is unpredictable and likely during a normal daily routine, sampling at a period shorter than the response time of the thermocouple would be give no additional information, would incur additional power consumption and would result in in an increased risk of inaccurate measurement.

\section{Conclusions}

The incorporation of power cycling and the reduction of processor clock speed can lead to a reduction in the power consumption of data acquisition equipment. However, reducing clock speed will increase the start-up and dataacquisition times, increasing the duty cycle, D. When using thermocouple based temperature sensors, the shortest sampling period can be determined from the consideration of the maximum temperature swing and the required accuracy.

\section{References}

[1] [online]. http://www.pewinternet.org/fact-sheets/health-factsheet/ (Nov. 2014).

[2] V.Chan, P.Ray, and N. Parameswaran, "Mobile eHealth monitoring: an agent-based approach," IET Communications, vol.2, no.2, pp.223-230, Feb. 2008.

[3] H.H. Asada, P. Shaltis, A. Reisner, Rhee Sokwoo, R.C. Hutchinson, "Mobile monitoring with wearable photoplethysmographic biosensors," Engineering in Medicine and Biology Magazine, IEEE, vol.22, no.3, pp.28,40, May-June 2003. 
[4] [online].

http://batteryuniversity.com/learn/article/the_high_po wer_lithium_ion/ (Nov. 2014).

[5] Kim, Kyungseok, and Vishwani D. Agrawal. "Ultra low energy CMOS logic using below-threshold dualvoltage supply." Journal of Low Power Electronics Vol. 7, no. 4 pp.460-470, 2011.

[6] Xiaoming Zhao, Ding-Yu Fei, Charles R. Doarn, Brett Harnett, and Ronald Merrell, "A Telemedicine System for Wireless Home Healthcare Based on Bluetooth ${ }^{\mathrm{TM}}$ and the Internet," Telemedicine Journal and e-Health, vol.10, no.2, pp.110-116, Nov. 2004.

[7] [Online]. http://www.bluetooth.com/Pages/what-isbluetooth-technology.aspx/ (Nov. 2014).

[8] Clark R, Edholm O. Man and His Thermal Environment. London: Edward Arnold; 1985.

[9] Houdas Y, Ring E. Human Body Temperature Its Measurement and Regulation. USA: Plenum Press; 1982.

[10] Frykberg R, Zgonis T, Armstrong D, Driver V, Giurini G, Kravitz S, et al. Diabetic Foot Disorders: A Clinical Practice Guideline (2006 Revision). The Journal of Foot and Ankle Surgery. 2006;45(5):1-66.

[11]N. Mathur, I. Glesk, and A.Buis, "Skin Temperature Prediction in Lower Limb Prostheses," IEEE Journal of Biomedical and Health informatics, in press, Nov. 2014. DOI: 10.1109/JBHI.2014.2368774 